\title{
A microfluidic electroporation device for cell lysis
}

\author{
Hang Lu, ${ }^{a c}$ Martin A. Schmidt ${ }^{b}$ and Klavs F. Jensen*c \\ Received 26th April 2004, Accepted 30th July 2004 \\ First published as an Advance Article on the web 5th November 2004 \\ DOI: 10.1039/b406205a
}

\begin{abstract}
We demonstrate a micro-electroporation device for cell lysis prior to subcellular analysis. Simple circuit models show that electrical lysis method is advantageous because it is selective towards plasma membrane while leaving organelle membrane undamaged. In addition, miniaturization of this concept leads to negligible heat generation and bubble formation. The designed microdevices were fabricated using a combination of photolithography, metal-film deposition, and electroplating. We demonstrate the electro-lysis of human carcinoma cells in these devices to release the subcellular materials.
\end{abstract}

\section{Introduction}

Analysis of proteins or organelles in biochemical and biomedical studies typically requires cell lysis (i.e., breaking open the cell membrane) in order to obtain subcellular materials. For many experiments, a total lysate is sufficient for the analysis (for example, intracellular proteins and RNA's); in other instances, subcellular fractionation is necessary and complete homogenization of cellular components is then undesirable. For example, cytochrome $c$ translocates from mitochondria to the cytosol in some apoptotic processes. ${ }^{1-3} \mathrm{~A}$ device that could rapidly separate the mitochondrial fraction from the cytosolic fraction would facilitate studies of the mitochondrial signal transduction pathways. Requirements for a device to perform cell lysis include speed (to prevent further biochemical changes), selectivity (breaking down cell membranes while protecting organellar membranes), and integration with other microfluidic devices. Examples of such integration could be upstream cell handling and manipulation (such as growth, stimulus, and cell sorting), downstream organelle purification and lysis, and protein separation and identification.

Conventionally cells can be lysed by either chemical or mechanical means. ${ }^{4}$ Because the cell membrane consists of a lipid double-layer, lysis buffers containing surfactants can solubilize the lipid membranes. The aim of such lysis is to release all intracellular and membrane proteins for gel-based separation processes (e.g. Western blots, and SDS-PAGE), ${ }^{5}$ which allow identification of specific proteins. Consequently in many of these processes, the organelle membranes are also dissolved. Therefore, chemical methods are generally not suitable for cell lysis when organelle integrity is critical. Additionally, removal of surfactant remaining in the sample after lysis could require additional separation steps. A second common method of cell lysis relies on mechanical breakdown of the cell membrane by shear and wear. For example, the Dounce Homogenizer uses a mortar-pestle setup where cell suspension is sheared between the two parts of the device. In a recent study, a miniaturized mechanical lysis system demonstrates complete homogenization of cells in order to release

*kfjensen@mit.edu subcellular proteins. ${ }^{6}$ While the efficiency of lysis is high, mechanical lysis does not provide the discrimination needed for organelle analysis. Other types of mechanical breakage such as ultrasonic homogenization also yield homogenized lysate in which organelles are broken. Lastly, thermal energy can also be used to lyse cells such as bacteria, yeasts, and spores. Heating disrupts the cell membrane and the intracellular materials are released. However, this method is only compatible with nucleic acids analysis because protein molecules denature in the course of heating. In order to enable subcellular fractionation in microfluidic systems without the complications of chemical and mechanical lysis, we have chosen to implement electroporation. This method has the potential for achieving rapid and selective cell lysis and is compatible with other downstream processes such as organelle separation by free flow electrophoretic techniques. $^{7}$

Electroporation is a common method for creating transient holes in cell membranes to deliver genetic material or smallmolecule therapeutics. ${ }^{8,9}$ External electric field induces changes in plasma membrane and disrupts the transmembrane potential. The microfluidic electroporation devices in this work uses extreme conditions where the stability of the cell membranes is permanently disrupted, holes on the cell membranes sustained, and intracellular materials released. The externally applied field is chosen to exploit differences in transcellular-membrane potential $(\sim 60 \mathrm{mV})$ and transorganelle-membrane potential $(\sim 160 \mathrm{mV}$ for mitochondria for example) so that the cell membrane is compromised with minimal impact on organelles, such as the mitochondria.

Prior work in micro-scale electroporation includes devices for the release of DNA and proteins, ${ }^{10,11}$ for gene delivery, ${ }^{12-14}$ and for monitoring cell viability. ${ }^{15}$ Many of these devices use pulsed electric fields and operate in noncontinuous mode. We present a continuous device that provides a periodic spatial change in electric field strength, which through the fluid motion translates to a pulsating field experienced by the cells. Advantages of this approach include continuous operation and tunable operating conditions for speed and selectivity of the lysis, as well as the potential for integration with upstream and downstream microfluidic components. 


\section{Experimental}

\subsection{Modeling cell lysis and designing devices}

Cells consist of a lipid double-layer membrane with intracellular organelles and cytosolic material. To a first approximation, cells interacting with an external electric field can be modeled using an equivalent $R C$ circuit. $^{8,9,16}$

Completing a circuit analysis gives the following equations for the transmembrane potential for the cells (eqn. (1)) and for organelles (eqn. (2)), ${ }^{9,16}$ where $E$ is the external field strength, $R$ the radii of particles, $C$ the capacitance of the memebranes per unit area, $r$ resistivity, and $\omega$ the frequency of the applied electric field. The subscript int denotes intracellular, org denotes organelle, a for the extracellular electrolyte, i for intracellular electrolyte (cytoplasm), and s for intra-organelle electrolyte. The parameters for the model are extracted from the literature. ${ }^{8,16-20}$

$$
\begin{gathered}
V=\frac{1.5 E R}{\left(1+I \omega R C\left(r_{\mathrm{i}}+r_{\mathrm{a}} / 2\right)\right)} \\
V_{\text {org }}=\frac{1.5 E_{\text {int }} R_{\text {org }}}{\left(1+I \omega R_{\text {org }} C_{\text {org }}\left(r_{\mathrm{s}}+r_{\mathrm{i}} / 2\right)\right)} \\
E_{\text {int }}=\frac{1.5 E}{1+\left(1 /\left[I \omega R C\left(r_{\mathrm{i}}+\left[r_{\mathrm{a}} / 2\right]\right)\right]\right)}
\end{gathered}
$$

\subsection{Microfabrication of electroporation devices}

The farication process is shown schematically in Fig. 1. First, Pyrex 7740 wafers (Bullen Ultrasonic Inc., Eaton, OH) were cleaned in a bubbling piranha solution (1 : 3 hydrogen peroxide in sulfuric acid) for $10 \mathrm{~min}$. Caution: the mixing process is strongly exothermic and releases hot vapor, and the mixture is highly reactive. Personal protective wares including face shield, apron, and gloves should be used. The cleaned wafers were rinsed in deionized water and dried in a wafer spin dryer. Once treated with hexamethyldisilazane (HMDS) vapor, the wafers were coated with image reversal resist AZ5214E (Clariant Corp.). Standard photolithography techniques (brief exposure through a mask followed by postexposure bake and a flood exposure) was used to pattern the resist layer. A thin film of gold $(200 \mathrm{~nm})$ with titanium $(10 \mathrm{~nm})$ as adhesion layer was deposited in an electron-beam evaporator. The metal seed-layer pattern was formed in a lift-off process whereby metal film on the photoresist was removed along with the resist in solvent.

Subsequently, a layer of a photo-patternable epoxy SU-8-50 (MicroChem Corp.) was spun and patterned using photolithography to create the trenches in which the gold electrodes were to be formed. The current supplied to the substrates was used to control the gold deposition rate; generally, to plate $50 \mu \mathrm{m}$-thick electrode required $4-5 \mathrm{hr}$ at $60{ }^{\circ} \mathrm{C}$ using a noncyanide-based plating solution (Technic Inc.). At the completion of electroplating, the SU-8 mold was removed in a Nanostrip solution. The wafers were rinsed and dried before a further cleaning step in oxygen plasma for at least $30 \mathrm{~min}$. Another layer of SU-8-50 was coated and patterned to form

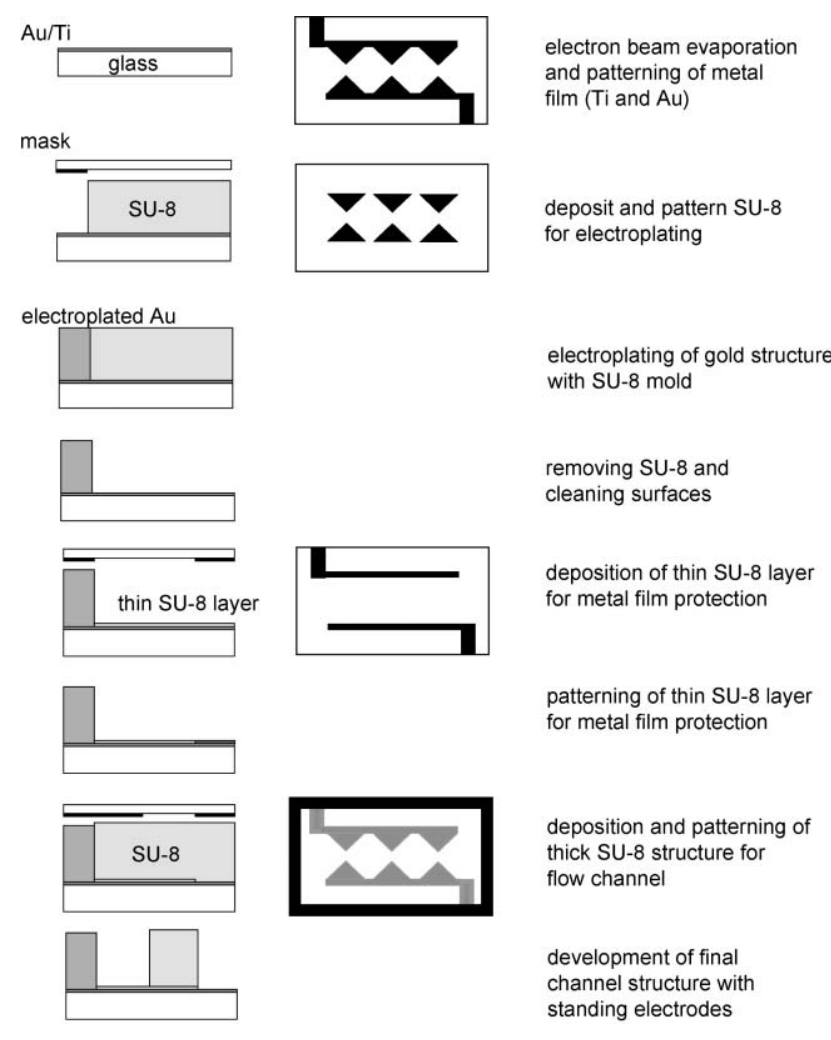

Fig. 1 Fabrication process for 3-D electrodes in microfluidic channels for cell lysis by electroporation.

the flow channels of the devices. Before being diced into individual devices using a Disco dice saw, the wafers were coated with a layer of thick resist AZ4620 (Clariant Corporation) to protect the features. Fluidic access holes on the glass substrate were drilled using a $0.75-\mathrm{mm}$ diamond drill bit. Solvents (acetone, ethanol, followed by isopropanol) were used to remove the thick photoresist. The device was capped by a piece of microscope cover-slip, glued by epoxy on the edges. Conductive epoxy (SPI Supplies) was used to bond wires to the thin-film gold contact pads on the glass substrate.

\subsection{Cell culture and microscopy}

HT-29 cells (human colon carcinoma, ATCC) were cultured in McCoy's 5A medium supplemented with $10 \%$ fetal serum, 100 units $\mathrm{ml}^{-1}$ penicillin, $100 \mu \mathrm{g} \mathrm{ml}^{-1}$ streptomycin and $2 \mathrm{mM}$ glutamine (Life Technologies) at $37{ }^{\circ} \mathrm{C}$ and $5 \% \mathrm{CO}_{2}$. The medium was changed every 2-3 days with cell-split ratio of $1: 5$ or $1: 8$. The cells were harvested with $0.25 \%$ trypsin (Life Technologies). The final cell suspension was $10^{5}-10^{6}$ cells ml $\mathrm{ml}^{-1}$ in concentration in the experiments. The fluorescence microscopy was performed with a Zeiss Axiovert-200 fluorescence microscope and images taken with an ORCA-100 cooled digital camera (Hamamatsu). A vital dye (final concentration of $5 \mu \mathrm{g} \mathrm{ml}^{-1}$ acridine orange and $5 \mu \mathrm{g} \mathrm{ml}^{-1}$ propidium iodide in water, Molecular Probes, Inc.) was added to harvested cells at room temperature and was readily taken up by the cells in $5 \mathrm{~min}$ (verified by fluorescence microscopy).

The devices were first sterilized with $2 \mathrm{ml}$ of ethanol solution $(70 \%)$, and then rinsed with $2 \mathrm{ml}$ of DI water followed by $1 \mathrm{ml}$ 
of PBS buffer. Lastly, $1 \mathrm{ml}$ of filtered bovine serum albumin in water $(2 \mathrm{wt} . \%)$ was flown through the device and incubated at room temperature for at least $20 \mathrm{~min}$ prior to the experiment to reduce adsorption of biological materials to the device.

Immediately after harvesting and staining the cells, $1 \mathrm{ml} \mathrm{BD}$ syringes were used to dispense the cells into two identical devices at a rate of $0.25-1 \mu \mathrm{min}^{-1}$ by a syringe pump (PHD2000, Harvard Apparatus). Voltage was controlled by a BK Precision $10 \mathrm{MHz}$ Sweep Function Generator (Model 4017). The experiments were performed under a variety of frequency and voltage conditions along with control experiments where cells were passed through the micro device with no voltage supply.

\section{Results and discussion}

\subsection{Modeling}

The most obvious choice for selective electroporation is to use a direct current (DC) electric field since the cell membrane would experience the largest applied transmembrane potential and the subcellular organelles none. However, the large electric field required to achieve lysis would result in a voltage exceeding the water electrolysis threshold $(\sim 1 \mathrm{~V})$, even in micro-scale devices. Thus, a DC field would inevitably create bubbles (hydrogen and oxygen gases), as well as extreme $\mathrm{pH}$ conditions near the electrodes.

To avoid such adverse conditions in microfluidic electroporation devices, an alternating current (AC) electric field is employed. Water electrolysis is minimized under a rapidly alternating field. Using representative values of material electrical properties, the modeling results shown in Fig. 2 demonstrate general trends of the transmembrane potentials. At very large frequencies, the potentials for both cells and subcellular particles drop off to a negligible magnitude, making the cells and organelles effectively transparent to the electric field. At intermediate frequency ranges, the trans-cellmembrane potential increases whereas the trans-organellemembrane potential drops as the frequency increases. Thus, there is an optimal range of operating frequency and voltage such that the trans-membrane potential is sufficient to break the cell membrane but not able to affect organelle membranes. The simple circuit model employed here is useful to guide the selection of experimental conditions, but it is not sufficient to predict the actual lysis performance.

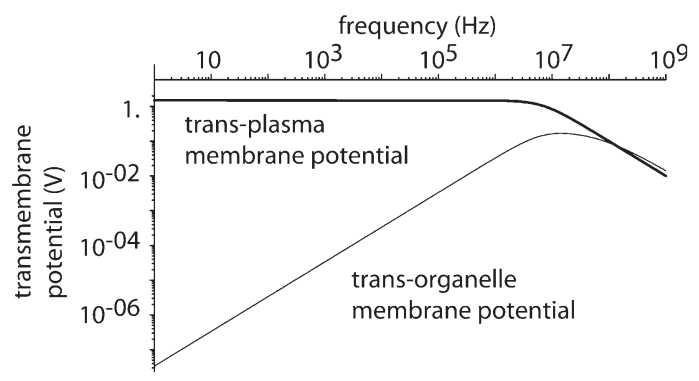

Fig. 2 Modeling results for external applied trans-membrane potentials for cells and sub-cellular organelles.

\subsection{Designing an electroporation microdevice}

Electroporation in microfluidic channels has several advantages. The voltages required to achieve cell lysis are much lower compared to macroscopic systems. By shrinking the inter-electrode distance to a few tens of microns, it is possible to reduce the voltage requirement to a few volts (three orders of magnitude smaller than typical voltages required in a macroscopic apparatus). Consequently, the power consumption becomes six orders of magnitude smaller and heat generation is minimal.

3.2.1. Design considerations. Conventional electroporation devices use batch processes in which electrical pulses are provided to cell suspensions in the electroporation chamber ${ }^{17,20-22}$ The batch format is difficult to integrate with other microfluidic devices and long residence time increases the possibilty of biological entities adhering to chamber surfaces. Therefore, we have chosen to design a continuous flow device in which periodic variation in electric field strength is due to the undulating geometry of the electrodes (Fig. 3). Moreover, the duration of electrical stimulation is controlled by the flow rate of the fluid carrying the suspended cells. The choice of inter-electrode distance was governed by practical considerations. The average size of mammalian cells (e.g. HT-29, a human colon carcinoma cell line used in this work) is on the order of $10 \mu \mathrm{m}$ in suspension. To avoid cells obstructing the flow path, the inter-electrode distance was set to be at least three cell diameters. The overal channel is $1100 \mu \mathrm{m}$ wide and contains four sets of microelectrode arrays (180 saw-teeth each).

3.2.2. Electric field strength calculation and heat effects. A field calculation solving Maxwell's equations in FEMLAB (Comsol, Inc.) using the peak-to-peak voltage (Fig. 4) reveals, as expected, that the electric field is non-uniform and highest near the tip of the electrodes. Moreover, the magnitude of electric field is in the range of $\mathrm{kV} \mathrm{cm}^{-1}$ while the AC voltage required is smaller than $10 \mathrm{~V}$.

Heat generation is a concern when performing electroporation in the conventional systems, but the six orders of

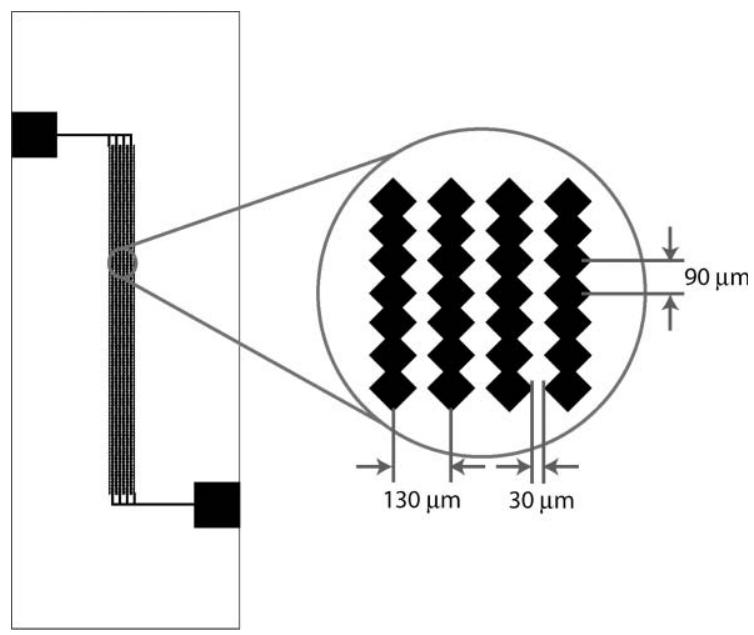

Fig. 3 Details of the saw-tooth design for micro-electroporation. 


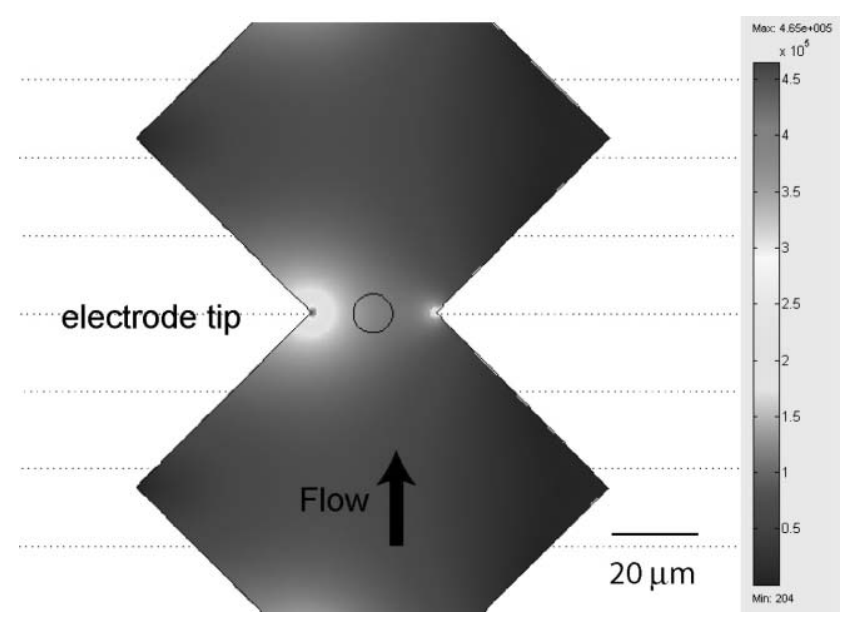

Fig. 4 Peak to peak voltage (in $\mathrm{V} \mathrm{m}^{-1}$ ) calculation in the microelectroporation device.

magnitude reduction in power levels and large surface area to volume ratios associated with microfluidic devices eliminate the effect. For example, an analysis of Joule heating for the device leads to a maximum temperature rise of less than $1 \mathrm{~K}$ (Fig. 5). In this simulation, the Fourier equation of heat conduction is solved with the following boundary and volume condition: all four edges of the device are in contact with room temperature $(300 \mathrm{~K})$ air, and the fluid conduit has a uniform volumetric heat generation rate of $0.1 \mathrm{~W}$ (assuming complete conversion of $10 \mathrm{~V}$ and $10 \mathrm{~mA}$ electrical energy to heat). The heat transfer in the fluid conduit is simplified to include only heat conduction; in reality, heat transfer also includes the heat carried away by the flowing fluids. Therefore the simulation is a conservative estimate of the temperature in the device.

3.2.4. Design layout and fabrication. Fig. 6 shows a schematic of the designed device. The electrodes were electrodeposited from the bottom metal seed layer to channel thickness $(\sim 50 \mu \mathrm{m})$. The advantages of having vertical electrodes

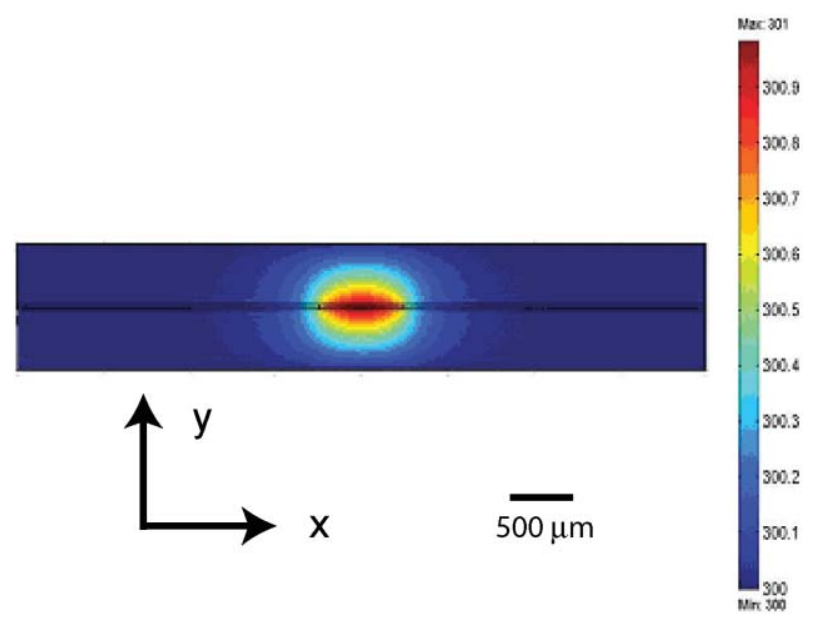

Fig. 5 FEMLAB simulations of heat generation and transfer in the micro-electroporation device for an applied voltage of $10 \mathrm{~V}$ and a current of $10 \mathrm{~mA}$. A cross section of the device is shown where the flow direction is out of the page.

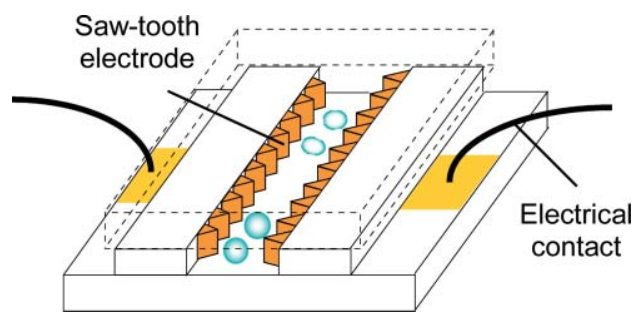

Fig. 6 Schematics of a micro electroporation device for cell lysis. Only one set of electrodes is shown for simplicity.

instead of planar electrodes include uniform electric field distribution and thicker electrodes that last longer in corroding environment. ${ }^{23}$ Photographs of the patterned metal layer with SU-8 mold for electroplating and the plated electrodes are shown in Fig. 7.

\subsection{Electroporation of HT-29 cells}

To demonstrate cell lysis in the micro-electroporation devices, we chose two lysis conditions, both within the intermediate frequency range $(1 \mathrm{kHz}-100 \mathrm{kHz})$. Suspensions of cultured HT-29 cells were characterized using microscopy techniques (both with phase contrast and with fluorescence staining). The cells from the electroporation experimental population appeared qualitatively different in physical characteristics from the control population that only experienced shear stresses by flowing through the devices. In transmission microscopy immediately after electroporation, treated cells had different morphology: they were more wrinkled, less rounded, and with much less defined membranes (Fig. 8). Cell fragments were also observed. Many of the ghost cells generally appeared smaller than normal healthy cells, indicating that subcellular materials had been released to the extracellular medium.

A vital stain with acridine orange and propidium iodide was used further to examine the effect of the electroporation on cell populations. Acridine orange (visible in green fluorescence channel) readily stains any cell. In contrast, healthy cells with intact membranes exclude propidium iodide (visible in the redorange fluorescence channel) from entering intracellular spaces, while cells with compromised membrane cannot discriminate against this stain. In the acridine orange stain (Fig. 9), the control population is much brighter than both experimental groups, suggesting that electroporation induces leakage through membranes and release of subcellular

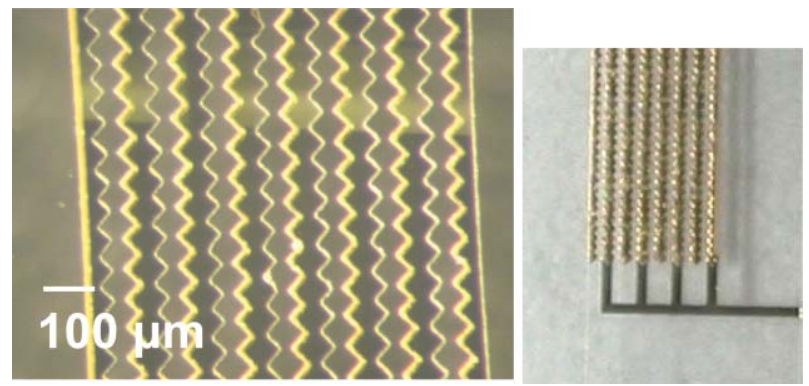

Fig. 7 Pictures of devices in fabrication processes: (left) after metallization and electrode-mold formation; (right) after electroplating. 

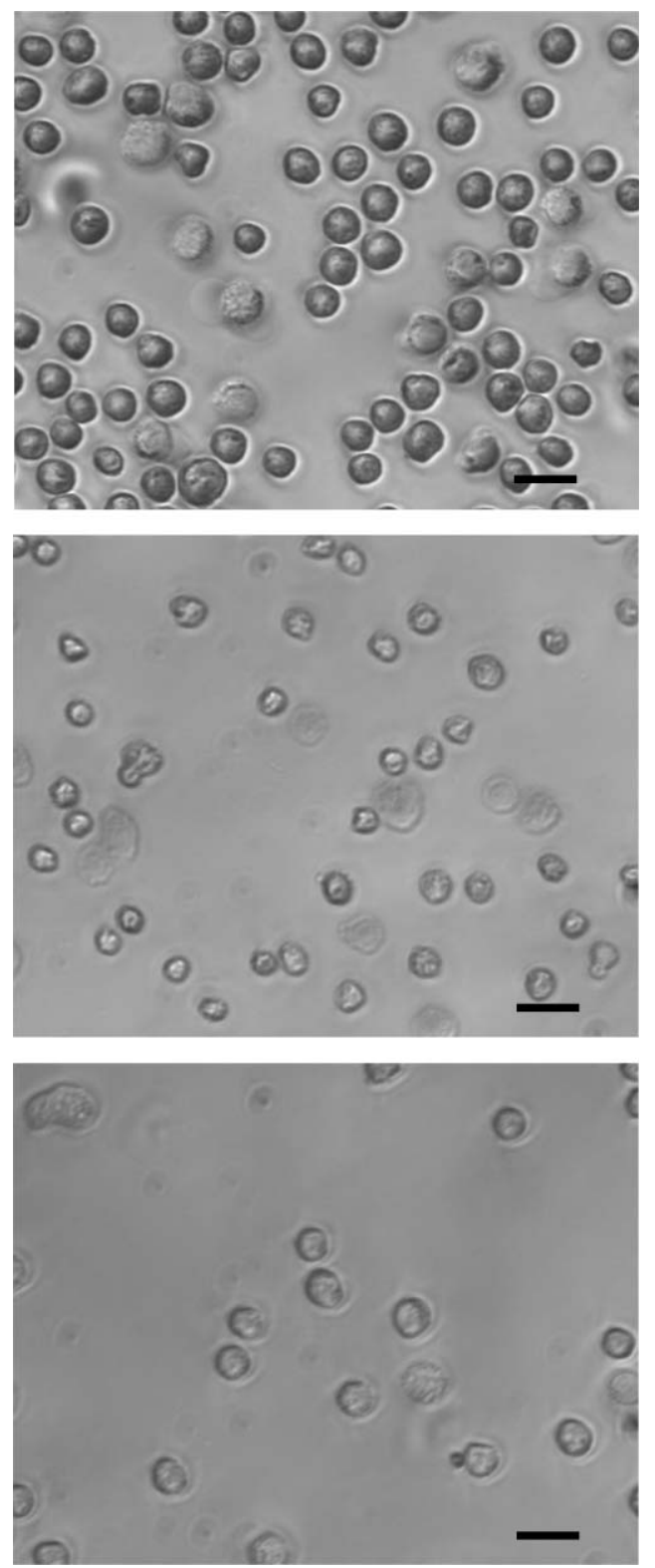

Fig. 8 Phase-contrast microscopy of cells that were treated by electroporating conditions in comparison to the control population: (top) control population; (middle) cells treated at $5 \mathrm{kHz}$ and $6 \mathrm{~V}$; (bottom) cells treated at $10 \mathrm{kHz}$ and $8.5 \mathrm{~V}$. Scale bars are $15 \mu \mathrm{m}$.

materials (therefore lower concentrations of acridine orange intracellularly after electroporation). In the propidium iodide stain (Fig. 10), the control population has a small fractions of cells that appear permeable to propidium iodide, due to a combination of normal and mechanically induced cell death. Electroporated cells are generally brighter, indicating that cell membranes are compromised and intracellular concentrations of propidium iodide are higher.

The modeling results earlier suggests that larger transmembrane potentials are achieved at lower frequency or higher applied peak-to-peak voltage. In general, cells that are treated with lower frequency have more permeable membranes, while cells treated with higher applied voltage are more likely to have undergone complete lysis. Cell lysis efficiency was quantified
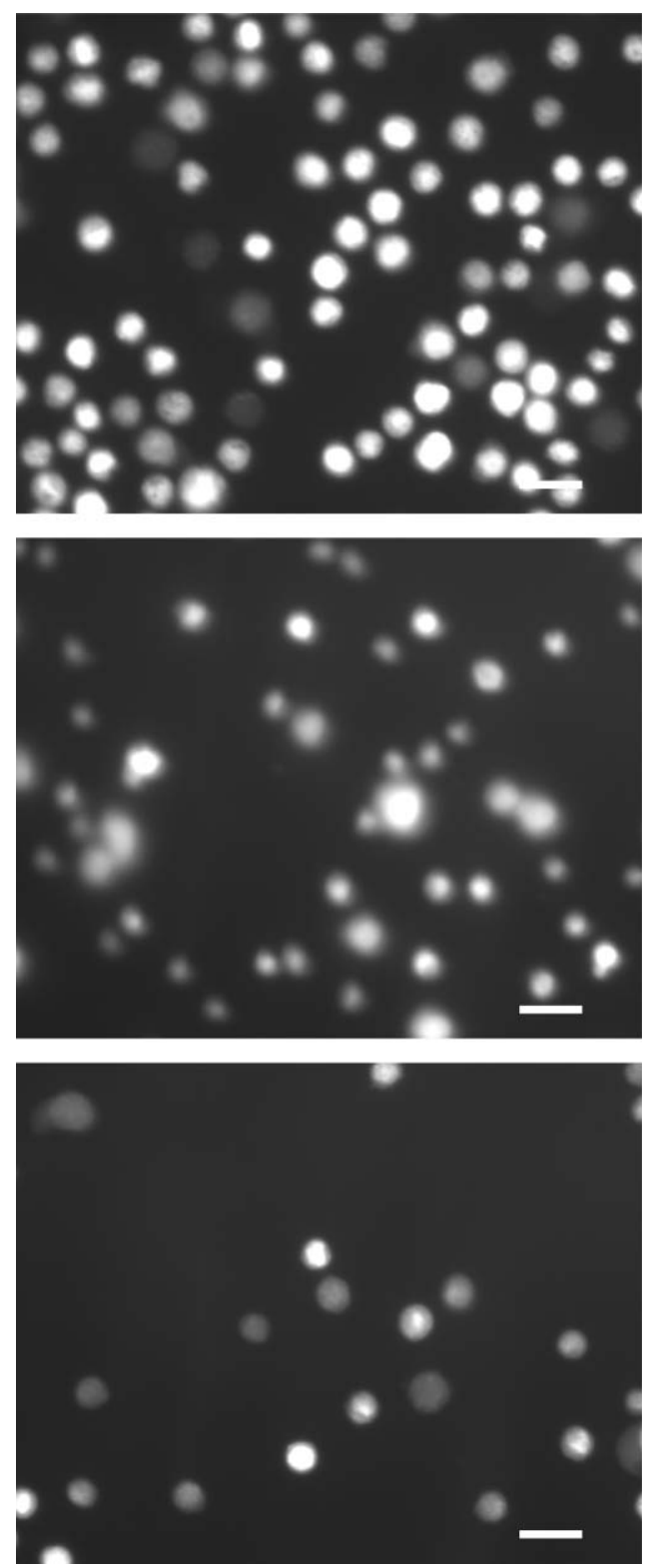

Fig. 9 Fluorescence microscopy results (green channel: excitation maximum $=485 \mathrm{~nm}$, emission maximum $=530 \mathrm{~nm}$ ) of electroporated cells and control cells: (top) control population; (middle) cells treated at $5 \mathrm{kHz}$ and $6 \mathrm{~V}$; (bottom) cells treated at $10 \mathrm{kHz}$ and $8.5 \mathrm{~V}$. Scale bars are $15 \mu \mathrm{m}$.

with samples collected at the end of the experiments. Table 1 lists the percentages of lysed cells and cells with compromised membranes at the end of the experiments for typical electroporation conditions. All samples started with the same cell density. The total cell count in each experiment was compared to that of the control population to calculate percentages of cells completely lysed in the electroporation device. The fraction of ghost cells after electroporation was quantified. These cells lost some organelles and cytosolic material during electroporation, but their cell membranes did not disintegrate completely and instead closed to form a particle. These particles were generally smaller and their membranes had higher contrast to the fluids than normal 

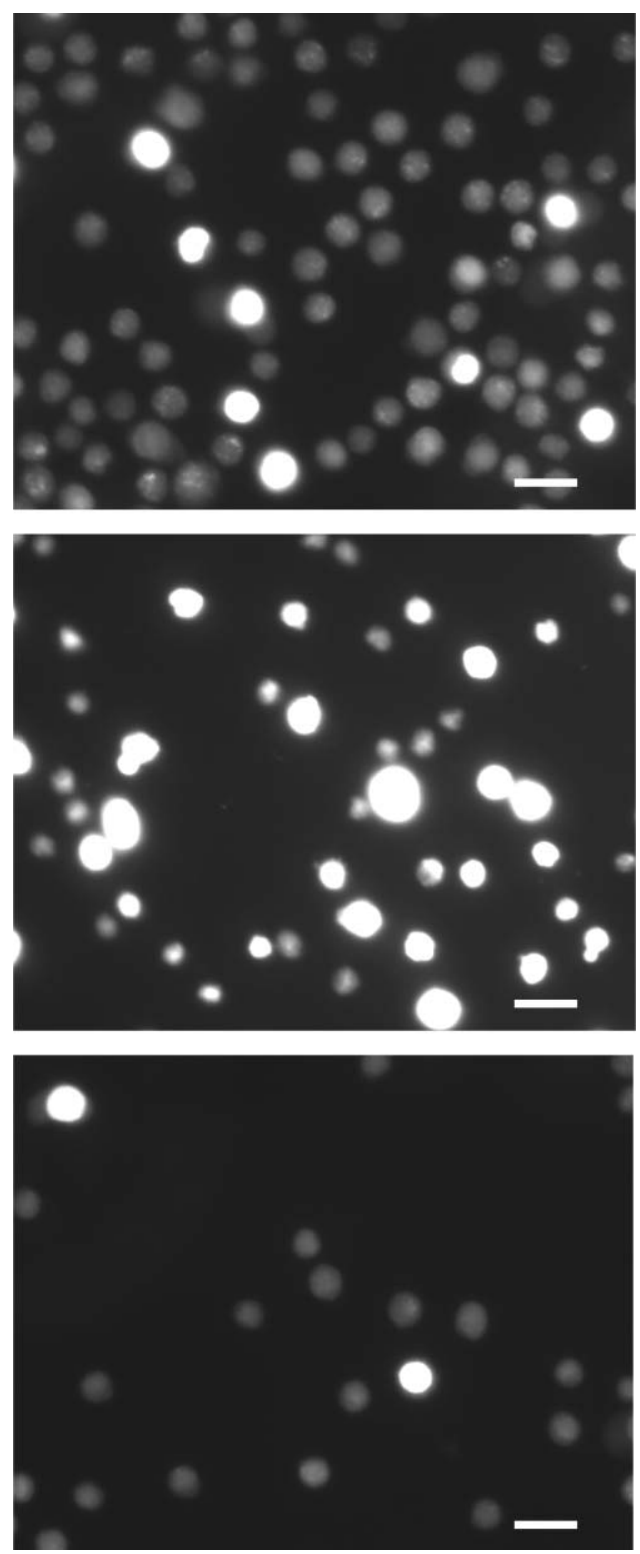

Fig. 10 Fluorescence microscopy results (red channel: excitation maximum $=535 \mathrm{~nm}$, emission maximum $=590 \mathrm{~nm}$ ) of electroporated cells and control cells: (top) control population; (middle) cells treated at $5 \mathrm{kHz}$ and $6 \mathrm{~V}$; (bottom) cells treated with $10 \mathrm{kHz}$ and $8.5 \mathrm{~V}$. Scale bars are $15 \mu \mathrm{m}$.

cells. The fractions of lysed cells and ghost cells were high, and combined numbers indicate that the majority of cells did not survive the electroporation process. The lower frequency treatment had a high percentage of ghost cells and lower lysed

Table 1 Comparison between cells treated under different electroporation conditions

\begin{tabular}{lcc}
\hline & $\begin{array}{c}\text { Cells treated at } \\
5 \mathrm{kHz}, 6 \mathrm{~V}\end{array}$ & $\begin{array}{c}\text { Cells treated at } \\
10 \mathrm{kHz}, \sim 8.5 \mathrm{~V}\end{array}$ \\
\hline $\begin{array}{l}\text { Fraction lysed calculated } \\
\text { from total cell count }\end{array}$ & $28 \%$ & $74 \%$ \\
Fraction of ghost cells & $81 \%$ & $71 \%$ \\
\hline
\end{tabular}

fraction, suggesting that this condition was gentler and perhaps more suitable for selective lysis of plasma membrane. Generally, optimal lysis of different cell types can be achieved by fine-tuning the operating frequency, voltage, the flow rate of the cell suspension (hence the residence time and the effective electrical pulse width), and the buffer conditions, particularly when intact or metabolically active organelles are desired.

Higher magnification of microscopy revealed a variety of cell membrane behavior, including swelling (Fig. 11a), lysis (Fig. 11b), morphological changes (membrane extrusion in particular, Fig. 11c), and rare electrofusion of two cells (Fig. 11d). These phenomena have been observed and modeled in macro scale electroporation procedures. ${ }^{8,19,20}$ One could imagine using the same micro-devices for electrofusion and molecular delivery. The electroporation conditions (e.g. field strength, duration of high voltage application, and the ionic strength and osmolarity of the buffer solution) could be altered to meet specific requirement for effective poration or lysis.

Dielectrophoresis (DEP) was observed as a consequence of the saw-tooth shapes of the micro electrodes and the resulting non-uniform electric field. Cell samples were focused in the flow stream at the positions corresponding to extrema of the electric field (Fig. 12). The particles in the images appear elongated because the camera is not sufficiently fast to resolve the shape of particles at the experimental flow velocity. A potential application of this DEP effect could be an immediate downstream identification of cytosolic proteins. The advantage is that the DEP effect keeps the samples from being diluted by the carrying fluid. ${ }^{24,25}$ For low-abundance subcellular protein, this sample handling technique could prove important in detecting the released proteins.

\section{Conclusion}

We have designed a micro electroporation device aided by a quantitative understanding of the interactions between cells and an external electric field. The micro device is effective in lysing cells while operating at more advantageous conditions than conventionmal systems: small voltages and power consumptions, continuous flow, small sample volume, and

(a)

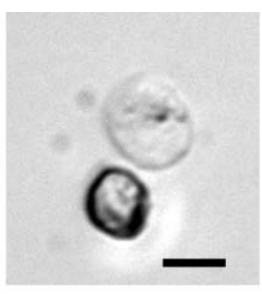

(c)

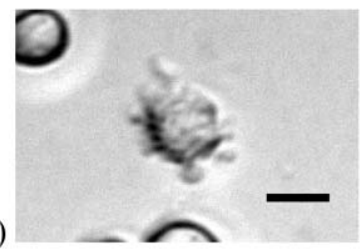

(d)

(b)

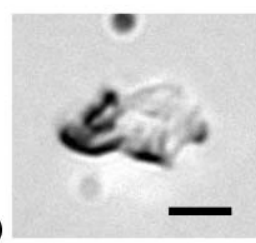

Fig. 11 Membrane behaviour in electric field-cell interactions observed in the micro electroporation devices. Scale bars are $10 \mu \mathrm{m}$. 


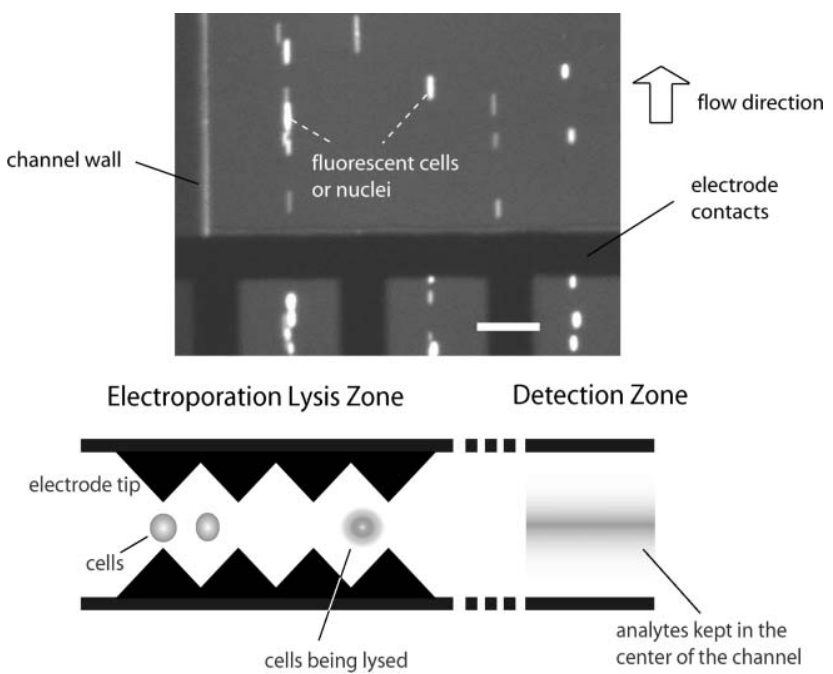

Fig. 12 (top) Dielectrophoresis effect observed in the flow channels. The image has been contrast-enhanced. (bottom) Schematics of the saw-tooth microelectrodes acting as a DEP device for focusing intracellular materials after electroporation. The scale bar in the top graph is $80 \mu \mathrm{m}$.

negligible heating. This device has the potential for integration with other microfluidic components as part of a lab-on-chip system for subcellular analysis, which would greatly facilitate large scale biochemical experimentation.

Hang Lu, ${ }^{a c}$ Martin A. Schmidt ${ }^{b}$ and Klavs F. Jensen*c

${ }^{a}$ Current, 513 Parnassus Ave., University of California, San Francisco, Department of Anatomy, CA 94143, USA

${ }^{b}$ Microsystems Technology Laboratories, MIT, 77 Massachusetts Ave. Cambridge, MA 02139, USA

${ }^{c}$ Department of Chemical Engineering, MIT, 77 Massachusetts Ave., Cambridge, MA 02139, USA. E-mail: kfjensen@mit.edu;

Fax: +1 617258 8224; Tel: +1 6172534589

\section{References}

1 E. Finkel, Science, 2001, 292, 5517, 624-626

2 C. Brenner and G. Kroemer, Science, 2000, 289, 5482, 1150-1151.
3 H. Li, S. K. Kolluri, J. Gu, M. I. Dawson, X. H. Cao, P. D. Hobbs, B. Z. Lin, G. Q. Chen, L. S. Lu, F. Lin, Z. H. Xie, J. A. Fontana, J. C. Reed and X. K. Zhang, Science, 2000, 289, 5482, 1159-1164.

4 J. M. Graham and D. Rickwood, Subcellular Fractionation: a Practical Approach, ed. D. Rickwood and B. D. Hames, Oxford University Press, New York, 1997, vol. 173.

5 T. E. Creighton, Protein Function: a Practical Approach, Oxford University Press, New York, 1997, vol. 175.

6 D. Di Carlo, K. H. Jeong and L. P. Lee, Lab Chip, 2003, 3, 4, 287-291.

7 H. Lu, S. Gaudet, M. A. Schmidt and K. F. Jensen, Anal. Chem., 2004, 76, 5705-5712.

8 E. Neumann, A. E. Sowers and C. A. Jordan, Electroporation and Electrofusion in Cell Biology, Plenum Press, New York, 1989.

9 J. C. Weaver, IEEE Trans. Plasma Sci., 2000, 28, 1, 24-33.

10 Y. Huang, K. L. Ewalt, M. Tirado, T. R. Haigis, A. Forster, D. Ackley, M. J. Heller, J. P. O'Connell and M. Krihak, Anal. Chem., 2001, 73, 7, 1549-1559.

11 Y. Huang and B. Rubinsky, Sens. Actuators, A, 2001, 89, 3, 242-249.

12 Y. C. Lin, C. M. Jen, M. Y. Huang, C. Y. Wu and X. Z. Lin, Sens. Actuators, B, 2001, 79, 2-3, 137-143.

13 Y. C. Lin and M. Y. Huang, J. Micromech. Microeng., 2001, 11, 5, 542-547.

14 Y. Huang and B. Rubinsky, Sens. Actuators, A, 2003, 104, 3, 205-212.

15 Y. Huang, N. S. Sekhon, J. Borninski, N. Chen and B. Rubinsky, Sens. Actuators, A, 2003, 105, 1, 31-39.

16 C. Grosse and H. P. Schwan, Biophys. J., 1992, 63, 6, 1632-1642.

17 R. P. Joshi and K. H. Schoenbach, Phys. Rev. E, 2000, 62, 1, $1025-1033$

18 S. Archer, T. T. Li, A. T. Evans, S. T. Britland and H. Morgan, Biochem. Biophys. Res. Commun., 1999, 257, 3, 687-698.

19 D. C. Chang, B. M. Chassy, J. A. Saunders and A. E. Sowers, Guide to Electroporation and Electrofusion, Academic Press, Inc., San Diego, CA, 1992.

20 P. T. Lynch and M. R. Davey, Electrical Manipulation of Cells, Chapman \& Hall, New York, 1996.

21 T. R. Gowrishankar and J. C. Weaver, Proc. Natl. Acad. Sci. USA, 2003, 100, 6, 3203-3208.

22 R. P. Joshi and K. H. Schoenbach, Phys. Rev. E, 2002, 66, 5, 52901.

23 R. P. Frankenthal and D. J. Siconolfi, J. Electrochem. Soc., 1982, 129, 6, 1192-1196.

24 S. Fiedler, S. G. Shirley, T. Schnelle and G. Fuhr, Anal. Chem., 1998, 70, 9, 1909-1915.

25 H. Morgan, D. Holmes and N. G. Green, IEEE Proc. Nanobiotechnol., 2003, 150, 2, 76-81. 